\title{
Kognitiv-psychoedukative Gruppenintervention bei stationären Patienten mit depressiven Erkrankungen - Ergebnisse einer prospektiven Pilotstudie
}

\author{
Annette Schaub Jana Kopinke Anke Neusser Marketa Charypar \\ Klinik für Psychiatrie und Psychotherapie, Ludwig-Maximilians-Universität München, Deutschland
}

\author{
Schlüsselwörter \\ Kognitive Verhaltenstherapie - Psychoedukation . \\ Depression - Affektive Erkrankungen - Stationäre \\ Gruppenpsychotherapie
}

\section{Zusammenfassung}

Hintergrund: Psychoedukation wird als zielorientierte und strukturierte Vermittlung krankheitsbezogener Informationen in psychiatrische und psychotherapeutische Ansätze integriert. Das kognitiv-psychoedukative Gruppenkonzept für stationäre Patienten mit affektiven Erkrankungen basiert auf der interaktiven Erarbeitung eines multidimensionalen, funktionalen Krankheitskonzepts, das Aspekte der Vulnerabilität, Belastungsfaktoren und Bewältigungsstrategien aufgreift. Es umfasst Psychoedukation über die Erkrankung und ihre Behandlungsmöglichkeiten, Aufbau positiver Aktivitäten, kognitive Umstrukturierung und Rezidivprophylaxe. Material und Methoden: Das therapeutische Vorgehen wurde an der Psychiatrischen Universitätsklinik München (LMU) entwickelt und modifiziert. Eine Machbarkeitsstudie wurde als Verlaufsmessung im "single group design» durchgeführt und Veränderungen wurden varianzanalytisch überprüft. 231 Patienten nahmen an 46 Gruppen teil. Ergebnisse: 125 Patienten bewerteten das Gruppenkonzept und das therapeutische Vorgehen. Es zeigte sich eine sehr hohe Akzeptanz der Gruppenintervention unter der pharmakologisch-psychotherapeutischen Behandlung: Mehr als drei Viertel der Patienten stuften diese als informativ, hilfreich und konkret umsetzbar ein. Schlussfolgerungen: Bereits im stationären Bereich können Patienten mit affektiven Erkrankungen von einem strukturierten Gruppenangebot profitieren, wenn dieses kognitive und motivationale Defizite berücksichtigt. Das didaktisch-therapeutische Vorgehen der Gruppenleiter, aber auch der Austausch der Betroffenen ist von zentraler Bedeutung. Im weiteren Verlauf wurde das Angebot für unipolare und bipolare Erkrankungen differenziert.

\author{
Key Words \\ Cognitive-behaviour therapy - Psychoeducational \\ interventions - Depression - Affective disorders · Inpatient \\ group-based treatment programme
}

\section{Summary}

A Cognitive-Behavioural and Psychoeducational Group Intervention for Depressed Inpatients - Results of a Prospective Pilot Study

Background: Psychoeducational interventions that provide disorder-related information in a goal-oriented and structured manner have been integrated in psychiatric and psychotherapeutic approaches. The present cognitive psychoeducational group programme for inpatients with affective disorders is based on a multidimensional functional illness concept which covers aspects of vulnerability, stressors and coping strategies. It covers information about the disorder and its treatment options, building up rewarding activities, cognitive restructuring and relapse prevention. Materials und Methods: This programme was developed and modified at the University of Munich, Department of Psychiatry (LMU). A feasibility study was set up in a follow-up single group design and analyses of variance (ANOVAs) were performed. A total of 231 patients participated in 46 groups. Results: 125 patients evaluated the effectiveness of the programme and its treatment strategies. The group programme was widely accepted among patients that were pharmacologically and psychotherapeutically treated: more than three quarters of the patients rated its contents to be informative, helpful and applicable to everyday living. Conclusions: Inpatients with affective disorders may already benefit from a structured group programme if it takes into account their cognitive and motivational deficits. The group leaders' didactic and psychotherapeutic strategies as well as the patients' exchanging ideas with each other play a central role. In the course of further investigations the programme was differentiated for patients with major depression or bipolar disorders.

\begin{tabular}{ll}
\hline KARGER & @ 2007 S. Karger GmbH, Freiburg \\
$\begin{array}{l}\text { Fax +49 76145207 14 } \\
\begin{array}{l}\text { E-mail Information@Karger.de } \\
\text { www.karger.com }\end{array}\end{array}$ & $\begin{array}{l}\text { Accessible online at: } \\
\text { www.karger.com/ver }\end{array}$
\end{tabular}




\section{Einleitung}

Depressive Erkrankungen gehören in der Bevölkerung [Bauer et al., 2004] und in stationären Behandlungseinrichtungen [Zielke, 1994] zu den häufigsten Diagnosen. Erschwerend für den Krankheitsverlauf sind die hohe Rückfallrate von 80-90\%, die hohe Komorbidität mit Angst-, Abhängigkeitsund Suchterkrankungen sowie die Suizidgefährdung [Hegerl, 2005]. Kognitiv-verhaltenstherapeutische, aber auch interpersonelle Ansätze haben in der Behandlung depressiver Patienten ihre Wirksamkeit belegt [de Jong-Meyer et al., 1996; de Rubeis et al., 2005; Hollon et al., 2005; Gloaguen et al., 1998]. Bereits in den 1980er Jahren beschrieben Lewinsohn und Mitarbeiter [1984; dt. Herrle und Kühner, 1994] ein Gruppenprogramm, das in aktuellen kognitiv-verhaltenstherapeutischen Therapiekonzepten aufgegriffen wird [Hautzinger, 2003]. Die Psychopharmakotherapie spielt bei schwerer Symptomatik und bei häufiger Chronifizierung eine wesentliche Rolle, da ihre zumeist gute symptomsupressive und rezidivprophylaktische Wirkung in placebokontrollierten Studien belegt wurde [Bauer et al., 2004]. In einer Übersicht zur stationären Depressionsbehandlung verweist Huber [2005] auf die höhere Effizienz der Kombinationsbehandlung gegenüber einer Pharmakotherapie bei schweren Depressionen [Keller et al., 2000] sowie unter Psycho- oder Pharmakotherapie nur teilremittierter Patienten [Paykel et al., 1999]. Stationäre Patienten werden zumeist mit multidimensionalen Therapiekonzepten behandelt [Wolfersdorf, 1997], die bislang kaum evaluiert wurden. Gruppeninterventionen [Backenstraß et al., 2001; Trautmann-Sponsel et al., 2000; Vieweg und Trabert, 2002; Pitschel-Walz et al., 2003] haben sich hinsichtlich Behandlungszufriedenheit und Symptomverbesserung im Prä-PostDesign und in der 6-Monats-Katamnese als wirksam erwiesen, jedoch fehlen kontrollierte Studien. Psychoedukative Gruppenansätze etablierten sich in der Behandlung von schizophrenen Erkrankungen [Pitschel-Walz et al., 2006], jedoch ist ihre Wirksamkeit bei depressiven Patienten noch relativ unerforscht [Risch und Stangier, 2006]. Der Stand der Forschung wird insgesamt als noch nicht zufriedenstellend gekennzeichnet, und das wissenschaftliche Niveau der Studien [Stuart und Bowers, 1995; Hegerl et al., 2004] sowie die Validität metaanalytischer Auswertungen [Jindal und Thase, 2003] werden methodenkritisch hinterfragt.

Seit 11 Jahren bietet die Klink für Psychiatrie und Psychotherapie der LMU München kognitiv-psychoedukative Gruppen für Patienten mit affektiven Erkrankungen an [Schaub et al., 2003, 2006], die Psychoedukation, Aktivierung, kognitive Umstrukturierung und Rückfallprävention umfassen. Kernstück der Psychoedukation ist das Vulnerabilität-Stress-Bewältigungs-Modell in einer für den Patienten verständlichen Version als Basis für ein «funktionales Krankheitsmodell», d.h. all jener Kognitionen, die dem Patienten helfen, seine Krankheit besser zu verstehen und sich bewusst zu werden, wie er diese positiv beeinflussen kann. Das oft inadäquate Krankheitskon- zept der Patienten, ihre Probleme bei der Krankheitsbewältigung, die mangelhafte Akzeptanz der medikamentösen Behandlung, die zum Teil eingeschränkten pharmakologischen Behandlungserfolge sowie die medikamentös weniger beeinflussbaren sozialen Defizite sind Indikationen für eine kombinierte Behandlung [Schaub, 2006]. Der Aufbau positiver Aktivitäten, das Erkennen und Modifizieren dysfunktionaler Kognitionen sowie der Umgang mit sich anbahnenden Krisen werden als Kompetenzen im Umgang mit depressiven Zuständen und ihren Exazerbationen gefördert.

Die nachfolgende Machbarkeitsstudie beschäftigt sich mit der Implementierung dieses Gruppenansatzes. Bei der Entwicklung und Evaluation des Behandlungskonzeptes für Depressionen sind wir folgendem Phasenmodell gefolgt: Phase I (1995-1997): Entwicklung bzw. Modifikation des Verfahrens; Erstellen eines vorläufigen Manuals; Schulung der Therapeuten und Behandlungsteams; Befragung der Patienten anhand eines Fragebogens bei Gruppenende zur Optimierung des Vorgehens. Phase II (1998-2000): (1) Überprüfung an einer klinischen Stichprobe im Prä-Post-Design und (2) Studie mit katamnestischen Erhebungen im naturalistischen Verlauf; Weiterentwicklung des Ansatzes und Entwicklung von Messinstrumenten (unter anderem Wissenstest, Fragebogen zum Krankheitsmodell). Phase III (2000-2005): Kontrollierte randomisierte Evaluationsstudie mit 1- und 2-Jahres-Katamnesen (Unterstützung durch das Bundesministerium für Bildung und Forschung). Die Ergebnisse der ersten Studie von Phase II werden hier berichtet. Diese untersucht, ob ein derartiges Konzept im stationären Bereich durchführbar ist und wie es von den Patienten bewertet wird. Anhand einer Teilstichprobe wird der Frage nachgegangen, ob diese Intervention in Kombination mit der Pharmakotherapie und dem Clinical Management zu einer Verbesserung von Befindlichkeit und Symptomatik beitragen kann.

\section{Entwicklung, Inhalte und Struktur des kognitiv- psychoedukativen Gruppenansatzes}

Da Ansätze eines derartigen Behandlungskonzeptes bereits in der Psychiatrischen Universitätsklinik Bonn entwickelt wurden, konnten diese aufgegriffen und an die vorliegenden Strukturen angepasst werden. Es galt, die stationsrelevanten Versorgungsansprüche zu berücksichtigen und die Integration in die Gesamtsituation zu optimieren. Die Anzahl der Therapiesitzungen wurde auf die durchschnittliche Verweildauer der Patienten abgestimmt. Mehrere depressionsspezifische Fortbildungsveranstaltungen für Ärzte, Psychologen und Pflegeteams wurden durchgeführt, um Wissensinhalte zu vermitteln und die Mitarbeiter im verhaltenstherapeutischen Vorgehen zu schulen. Verhaltenstherapeutische Aspekte wurden stärker in die klinische Versorgung integriert sowie patientenund interventionsbezogene Supervision angeboten, um spezifische Fragen (z.B. Indikation) zu klären und den Informa- 
Tab. 1. Beschreibung des Gruppenkonzeptes

\begin{tabular}{|c|c|}
\hline Sitzung & Thema \\
\hline \multicolumn{2}{|c|}{ Psychoedukation } \\
\hline 1 & $\begin{array}{l}\text { Vorstellung der Teilnehmer und des organisatorischen } \\
\text { Ablaufs } \\
\text { Darstellung des Behandlungskonzepts } \\
\text { Aufstellen und Erläutern der Gruppenregeln } \\
\text { Symptome und Anzeichen einer Depression }\end{array}$ \\
\hline 2 & $\begin{array}{l}\text { Ursachen und Behandlungsmöglichkeiten der Depression } \\
\text { Entwicklung des Vulnerabilitäts-Stress-Bewältigungs-Modells }\end{array}$ \\
\hline 3 & $\begin{array}{l}\text { Funktionsweise der Nervenzellen } \\
\text { Wirkungsweise der Antidepressiva, weitere Psychopharmaka } \\
\text { Nebenwirkungen und Selbsthilfestrategien } \\
\text { Weitere Behandlungsansätze, psychotherapeutische Ansätze }\end{array}$ \\
\hline \multicolumn{2}{|c|}{ Aktivitätenaufbau } \\
\hline 4 & $\begin{array}{l}\text { Das Teufelskreismodell der Depression } \\
\text { Die Depressionsspirale und die Anti-Depressionsspirale } \\
\text { Stimmungsprotokoll und Aktivitätenliste }\end{array}$ \\
\hline 5 & $\begin{array}{l}\text { Kriterien zur Planung positiver Aktivitäten } \\
\text { Wochenplan }\end{array}$ \\
\hline 6 & $\begin{array}{l}\text { Bedeutung des Gleichgewichts zwischen positiven Aktivitäten } \\
\text { und Anforderungen (Selbstverstärkerpläne) } \\
\text { Planung von Teilzielen und Anerkennungen }\end{array}$ \\
\hline \multicolumn{2}{|c|}{ Kognitive Verhaltenstherapie } \\
\hline 7 & $\begin{array}{l}\text { Einführung in die kognitive Verhaltenstherapie } \\
\text { Die kognitive Triade } \\
\text { Gedanken, Gefühle, Handlungen: das A-B-C-Schema } \\
\text { Protokollieren von automatischen Gedanken }\end{array}$ \\
\hline 8 & $\begin{array}{l}\text { Typische gedankliche Verzerrungen und deren Veränderung } \\
\text { Die Spaltentechnik zur Veränderung automatischer } \\
\text { depressiver Gedanken } \\
\text { Grübelstopp }\end{array}$ \\
\hline 9 & $\begin{array}{l}\text { Der Zusammenhang von automatischen depressiven } \\
\text { Gedanken und depressionsfördernden Grundüber- } \\
\text { zeugungen (Eisbergmodell) } \\
\text { Typische depressionsfördernde Grundüberzeugungen } \\
\text { Tagesprotokoll automatischer Gedanken und realistischer } \\
\text { Alternativen }\end{array}$ \\
\hline 10 & $\begin{array}{l}\text { Erkennen und Verändern der depressionsfördernden } \\
\text { Grundüberzeugungen } \\
\text { Zusammenfassung: Kognitive Verhaltenstherapie }\end{array}$ \\
\hline \multicolumn{2}{|c|}{ Rückfallprophylaxe } \\
\hline 11 & $\begin{array}{l}\text { Frühwarnsymptome } \\
\text { Krisenplan und medikamentöse Rückfallprophylaxe } \\
\text { Individuelle Rückfallprophylaxe }\end{array}$ \\
\hline 12 & $\begin{array}{l}\text { Umgang mit der Erkrankung nach außen } \\
\text { Literatur und Adressen, Abschluss }\end{array}$ \\
\hline
\end{tabular}

tionsaustausch zwischen den Behandelnden zu unterstützen. Parallel wurde auch mit dem Aufbau einer Depressionsspezialstation begonnen. Der Besuch einer etablierten Depressionsstation [Wolfersdorf, 1997] diente dem Motivationsaufbau und der Abklärung wichtiger Fragen (z.B. Umgang mit Suizid auf Station; Wolfersdorf et al. [2005]). Die Entwicklung der Gruppenintervention wurde zu Beginn hauptsächlich durch Mitarbeiter dieser Depressionsspezialstation unter- stützt und durch diese stationsintern durchgeführt. Anfänglich wurden die Inhalte auf drei Berufsgruppen aufgeteilt: Ärzte informierten über die Erkrankung und ihre Behandlung, das Pflegepersonal vermittelte Schritte zur Aktivierung und Psychologen kognitive Strategien im Umgang mit Depressionen. Da die Teilnehmer jedoch die konstante Präsenz der Gruppenleiter wünschten, leiteten in Folge zwei Psychologen in Psychotherapieausbildung die Gruppe, und ärztliche Kollegen übernahmen die Wissensvermittlung über biologische Aspekte und die Psychopharmakotherapie in zwei Sitzungen. Ein vorläufiges Manual und die entsprechenden Informations- und Arbeitsblätter für die Patienten wurden ausgearbeitet und regelmäßige wöchentliche Supervisionen durchgeführt. Das ursprünglich auf 8 Sitzungen angelegte verhaltentherapeutisch orientierte Gruppenprogramm wurde im Weiteren um Strategien zur Rezidivprophylaxe erweitert und umfasste als kognitiv-psychoedukative Gruppenintervention schließlich 12 Sitzungen (je 1,5 h, 2-mal wöchentlich) [Schaub et al., 2006].

Aufgrund der hohen Nachfrage wurden stationsübergreifende Gruppen angeboten. Die Gruppen wurden zu Beginn geschlossen geführt, um die Vertrautheit der Patienten untereinander zu gewährleisten. Da im Weiteren eine höhere Versorgungskapazität gewünscht wurde und dies besser durch offene Gruppen abdeckt werden kann, wurde das Behandlungskonzept in vier Behandlungsblöcke unterteilt; und jeweils zu Beginn der ersten drei Blöcke konnten erneut Patienten in die Gruppe aufgenommen werden. Parallel zu den patientenbezogenen Gruppen wurden Angehörigengruppen angeboten und entsprechende Materialien ausgearbeitet. Diese Gruppen umfassten anfänglich 8 Termine (1,5 h, alle 2 Wochen), wurden dann aber auf 2-3 Blöcke verkürzt. Im Gegensatz zu einigen bereits vorliegenden Ansätzen umfasst dieses Behandlungsangebot psychoedukative und kognitiv-verhaltenstherapeutische Elemente [Beck et al., 1994; Hautzinger, 2003]. Tabelle 1 veranschaulicht die Behandlungselemente.

Im Rahmen der Psychoedukation werden die Patienten ermutigt, über ihre Krankheitskonzepte zu sprechen, d.h. über ihre Annahmen hinsichtlich Ätiologie und Rezidive sowie über ihre Symptome und wie sie diese bewältigen. Da die Gruppenintervention die Selbstwirksamkeit [Bandura, 1977] der Patienten erhöhen soll, wird ihnen erläutert, wie sie durch eigene Bewältigungsstrategien und durch Mitarbeit in Bezug auf die Pharmakotherapie Einfluss auf die Symptomatik und den Krankheitsverlauf nehmen können. Die Aspekte Aktivierung, Gefühle und Denken und ihre wechselseitige Beeinflussung werden anhand eines Dreiecks veranschaulicht und mögliche Ansatzpunkte hervorgehoben. Der Patient wird angeleitet, positive Aktivitäten zu planen und durchzuführen sowie Selbstverstärkungspläne zu erarbeiten. Als Grundlage der kognitiven Umstrukturierung wird der Patient auf den $\mathrm{Zu}$ sammenhang zwischen Bewertungen und damit einhergehenden Gefühlen hingewiesen. Während des Tages immer wieder auftretende negative Gedanken werden dokumentiert. Die 
Tab. 2. Wesentliche Aspekte bei der Durchführung einer kognitivpsychoedukativen Gruppe

Individualisierung des Vorgehens, d.h. Verknüpfung der Inhalte mit individuellen Themen und Erfahrungen der Teilnehmer.

Anpassung an die Belastbarkeit, das kognitive Niveau und die emotionale Befindlichkeit der jeweiligen Gruppenteilnehmer.

Flexible Handhabung des Therapieleitfadens.

Einsatz von Cotherapeuten.

Aufklärung und Motivierung durch den behandelnden Arzt.

Angebot von Einzelgesprächen bei Patienten mit Schwierigkeiten bei der Teilnahme (z.B. bei wahnhaften Symptomen) sowie bei Themen, die sich nicht für die Gruppe eignen.

Weitergehende Gruppenteilnahme nach Wechsel ins ambulante Setting. Paralleles Angebot einer Angehörigengruppe.

ihnen zugrunde liegenden dysfunktionalen Annahmen (z.B. «Ich muss alles richtig machen, um anerkannt zu sein.») werden erarbeitet und hinterfragt. Der Umgang mit individuellen Frühwarnsignalen und mögliche Schritte der Rückfallprophylaxe sind wesentliche Aspekte, um sich anbahnende Krisen besser abfangen zu können. Tabelle 2 listet verschiedene Aspekte auf, die sich aus unserer Sicht für die Durchführung einer kognitiv-psychoedukativen Gruppe als hilfreich erwiesen haben.

\section{Patienten und Methoden}

\section{Patienten}

Patienten mit einer affektiven Störung (klinisches Interview gemäß ICD10-Kriterien) wurden, wenn sie den Einschlusskriterien entsprachen, über die Studie aufgeklärt und nach ihrer Einwilligung konsekutiv in die Studie eingeschlossen. Die Einschlusskriterien bezogen sich auf eine hinlängliche Belastbarkeit, 2-mal die Woche 60-90 min an einer Gruppenintervention teilzunehmen, hinreichende intellektuelle Fertigkeiten und Kenntnis der deutschen Sprache. Eine Altersbegrenzung wurde nicht vorgegeben. Aus schlusskriterien auf Achse I waren das Vorliegen organisch bedingter psychischer Erkrankungen, Psychosen aus dem schizophrenen Formenkreis, Komorbididät mit primärem Substanzmissbrauch bzw. Substanzabhängigkeit; auf Achse II Borderline- und antisoziale Persönlichkeitsstörungen. Ab 1999 wurden bipolare Patienten nicht mehr eingeschlossen. Gleichzeitige Behandlung mit Elektrokrampftherapie, Schlafentzug oder Lichttherapie waren keine Ausschlusskriterien. Die Einschlusskriterien waren sehr weit gefasst, da diese Intervention unter Versorgungsaspekten durchgeführt wurde. Das Studienprotokoll wurde von der Ethikkommission der Medizinischen Fakultät der Universität München 1998 genehmigt und entspricht den ethischen Standards der Deklaration von Helsinki in ihrer erweiterten Form von 1975 sowie ihren Zusätzen von 1983, 1989 und 1996 [World Medical Association declaration of Helsinki, 1997]. Die Patienten wurden mit Psychopharmaka behandelt, wie es in den entsprechenden Therapieleitlinien [Benkert und Hippius, 1998; DGPPN, 2000] empfohlen wird. Bei Gruppenbeginn wurden 78,8\% der Patienten mit Antidepressiva behandelt (Trizyklika: 50,9\%; SSRI: 23\%; die übrigen lagen unter $10 \%$ ), davon 25,7\% in Kombination mit Mood-Stabilizern bzw. 7,5\% in Kombination mit Neuroleptika. Bei Gruppenende wurden 83,6\% der Patienten mit Antidepressiva (Trizyklika: 55,8\%; SSRI: 23,9\%; Mirtazapin: $11,1 \%$; die übrigen lagen unter $10 \%$ ) behandelt, davon $26,1 \%$ in Kombination mit Mood-Stabilizern bzw. 14,6\% in Kombination mit Neuroleptika.
Tab. 3. Demografische und klinische Beschreibung der Stichprobe $(\mathrm{N}=231)$

\begin{tabular}{|c|c|c|c|}
\hline Kennwerte & $\mathrm{n}$ & M & SD \\
\hline \multicolumn{4}{|l|}{ Höchster erreichter Schulabschluss ${ }^{1}$} \\
\hline Keiner, ohne Qualifikation & 50 & & \\
\hline Hauptschulabschluss mit Qualifikation & 56 & & \\
\hline Mittlere Reife & 33 & & \\
\hline Hochschulreife & 75 & & \\
\hline \multicolumn{4}{|l|}{ Höchster erreichter Berufsabschluss ${ }^{1}$} \\
\hline Keiner & 45 & & \\
\hline Lehre, Fach-/Meisterschule & 123 & & \\
\hline Hochschule & 61 & & \\
\hline \multicolumn{4}{|l|}{ Berufliche Situation } \\
\hline Unbeschäftigt & 92 & & \\
\hline Beschäftigt & 131 & & \\
\hline Frauen & 138 & & \\
\hline Alter (in Jahren) & & 51,0 & 14,2 \\
\hline Erkrankungsdauer (in Jahren) & & 8,8 & 10,5 \\
\hline Anzahl bisheriger Hospitalisationen & & 2,6 & 2,5 \\
\hline \multicolumn{4}{|l|}{ Kumulative Hospitalisationsdauer zum } \\
\hline Zeitpunkt der Gruppe (in Wochen) & & 11,9 & 8,5 \\
\hline HAMD $(n=46)$ & & 18,4 & 7,7 \\
\hline MADRS $(n=80)$ & & 24,0 & 8,3 \\
\hline BDI $(n=62)$ & & 23,0 & 11,3 \\
\hline Wissenstest $(0-116)(\mathrm{n}=23)$ & & 84,6 & 15,9 \\
\hline
\end{tabular}

${ }^{1}$ Erhoben über den jeweils höchsten erreichten Schul- bzw. Berufsabschluss.

\section{Therapeuten}

Insgesamt führten 19 Gruppenleiter im Alter von durchschnittlich $\mathrm{M}=$ 34,3 Jahren (Range 27-48) die Gruppeninterventionen durch. Ein Drittel der Therapeuten befand sich noch am Beginn, zwei Drittel befanden sich im fortgeschrittenen Stadium einer verhaltenstherapeutischen Ausbildung. Die Zuweisung der Patienten auf die Therapiegruppen erfolgte nach Verfügbarkeit. Nach durchschnittlich jeder vierten Gruppensitzung fand zumeist eine videogestützte Supervision statt, um die Qualität und die Manualtreue der durchgeführten Therapien zu gewährleisten.

\section{Erhebungsinstrumente}

Es wurde ein Fragebogen zur Zufriedenheit mit der Gruppenintervention mit 14 Themenschwerpunkten erhoben, der ursprünglich für Psychosen [Schaub, 2003] entwickelt und für Depressionen modifiziert wurde. Er erfasst Interessen, Zufriedenheit und Befindlichkeit der Teilnehmer. In einer Subgruppe wurden Erhebungen zu Beginn und am Ende der Gruppenintervention zur Dokumentation des Therapieverlaufs durchgeführt. Die Hamilton Depression Scale [HAMD; Hamilton, 1986], die Montgomery Asberg Depression Scale [MADRS; Asberg et al., 1978; deutsch: Neumann und Schulte, 1989], das Beck-Depressionsinventar [BDI; Beck und Steer, 1987; deutsche Fassung: Hautzinger et al., 1995] beurteilten die depressive Symptomatik. Zudem wurde ein Multiple-Choice-Fragebogen zum Wissensstand über depressive Störungen mit 27 Fragen entwickelt.

\section{Statistische Verfahren}

Alle statistischen Berechnungen wurden mit Hilfe des Programmpakets SPSS 13.0 für Windows durchgeführt. Zur Testung auf statistische Unterschiede wurden t-Tests für abhängige Stichproben und Varianzanalysen mit Messwiederholung berechnet. 


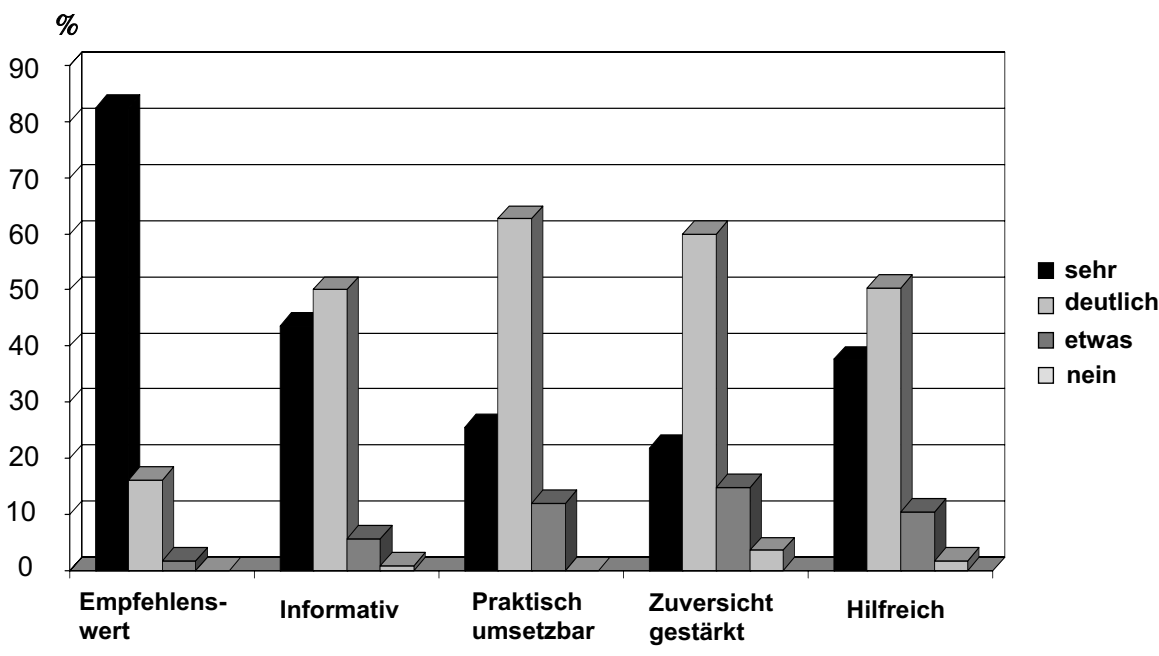

Gruppenintervention $(\mathrm{n}=125)$.

\section{Ergebnisse}

\section{Beschreibung der Stichprobe}

Im Rahmen der Implementierungsphase nahmen 360 affektiv erkrankte Patienten (220 Frauen, 140 Männer) an diesen stationären Gruppen teil. Diese Stichprobe bezieht sich auf 231 Patienten (138 Frauen, 93 Männer; 64,2\% der ursprünglichen Stichprobe), die ab 1998 in die Studie einwilligten. 140 Patienten $(60,6 \%)$ wurden auf einer speziellen Depressionsstation rekrutiert. Die Mehrzahl der Patienten litt unter depressiven Episoden (ICD-10 F32: $\mathrm{n}=107 ; 46,3 \%$ ) oder an einer rezidivierenden depressiven Störung (F33: $\mathrm{n}=81 ; 35,1 \%)$. Die übrigen Diagnosen waren anhaltende affektive Störung (6,5\%), Anpassungsstörung (2,2\%) und depressive Episode bei bipolarer Störung (9,9\%). Bei 45,0\% der Patienten wurde die Erkrankung als schwer eingestuft. $24,7 \%$ litten unter somatischen und $11,7 \%$ unter psychotischen Symptomen.

Hinsichtlich der klinischen und soziodemografischen Variablen zeigte sich ein heterogenes Bild (Tab. 3). 102 Patienten $(44,2 \%)$ waren zum ersten Mal in stationärer Behandlung. Die Erkrankungsdauer streute von $<1$ Jahr (12,1\%), 1-5 Jahre (40,7\%) bis zu 6-15 Jahre (23,8\%) und mehr. Das hohe Bildungsniveau entspricht der Klientel von Universitätskliniken. $\mathrm{Zu}$ Beginn des stationären Aufenthalts war etwa die Hälfte der Patienten unbeschäftigt. Erwartungsgemäß überwog der Anteil der Frauen mit 59,7\%. Die Depressionsskalen (HAMD, MDRS, BDI) sprechen für eine deutliche fremdund selbstbeurteilte Beeinträchtigung. Im Wissenstest erreichen die Patienten nur $73 \%$ der möglichen Punktzahl.

\section{Beurteilung des Gruppenangebots durch die Patienten}

Von 1998 bis 2000 nahmen insgesamt 231 Patienten an 34 Gruppen mit durchschnittlich 7 Patienten teil. Die geringe Abbrecherquote von 17,1\% deutet auf eine hohe Akzeptanz hin. Studienteilnehmer, die an den Posterhebungen teilnahmen, unterschieden sich nicht signifikant hinsichtlich klinischer und soziodemografischer Variablen von den anderen. Das Gruppenangebot wurde von den Patienten sehr positiv aufgenommen. Bei einer Rücklaufquote von $54 \%(\mathrm{n}=125)$ ergab sich die in Abbildung 1 aufgeführte Einschätzung des Gruppenangebots. Die Patienten, die den Fragebogen ausfüllten, unterschieden sich nicht in klinischen oder soziodemografischen Merkmalen von den anderen Patienten.

98,4\% der Patienten gaben an, dass sie die Gruppenintervention anderen Betroffenen empfehlen würden (Abb. 1). 99,1\% der Patienten schätzten das Verhalten der Gruppenleiter als kompetent ein. Besonderes Interesse hatten die Teilnehmer an Informationen über Entstehungsbedingungen und Verlauf (94,7\%), Symptome (92,0\%), Umgang mit der Erkrankung (92,0\%), Behandlungsmöglichkeiten (91,9\%), Maßnahmen zur Rezidivprophylaxe (91,0\%) sowie Nebenwirkungen und möglichen Gegenmaßnahmen (85,6\%). 95\% der Teilnehmer gaben an, dass sie mindestens zwei Drittel der Gruppenzeit anwesend waren. Der Aufwand für die regelmäßige Teilnahme wurde von $64,2 \%$ als erheblich eingestuft. Auf einer 5stufigen Ratingskala zur Befindlichkeit während der Gruppe lagen die Angaben durchgängig im Durchschnittsbereich, lediglich die Einstufung der Anspannung war im Grenzbereich.

\section{Akuter Behandlungsverlauf}

Bei einer Teilstichprobe war es möglich, das Ausmaß ihrer Depressivität vor und nach der Gruppenteilnahme zu erheben. Die ANOVAs ergaben, dass sich der Grad der selbst- (BDI) bzw. fremdberichteten (MADRS, HAMD) Depressivität höchst signifikant verbesserte (BDI, $\mathrm{n}=41$, prä: $\mathrm{M} \pm \mathrm{SD}=24,0$ $\pm 10,8$, post: $\mathrm{M} \pm \mathrm{SD}=15,9 \pm 9,6 ; \mathrm{F}=17,717, \mathrm{p}<0,001$; MADRS, $n=65$, prä: $\mathrm{M} \pm \mathrm{SD}=24,2 \pm 8,6$, post: $\mathrm{M} \pm \mathrm{SD}=15,2$ $\pm 9,8 ; \mathrm{F}=48,323, \mathrm{p}<0,001 ; \mathrm{HAMD}, \mathrm{n}=32$, prä: $\mathrm{M} \pm \mathrm{SD}=18,3$ $\pm 7,1$, post: $\mathrm{M} \pm \mathrm{SD}=11,8 \mathrm{M} \pm 8,2 ; \mathrm{F}=27,199, \mathrm{p}<0,001)$. Es gilt allerdings, die kombinierte antidepressive Behandlung zu berücksichtigen. Definiert man die Responserate als Therapieansprechen («response») im Sinne einer 50\%igen Reduktion des HAMD-Werts [orientiert an Frank et al., 1990] oder des BDIWerts nach 12 Sitzungen (6 Wochen), fallen 43,8\% bzw. 24,4\% der Patienten in diesen Bereich. In diesem Kontext gilt es zu erwähnen, dass etwa 56\% der Patienten auch nach Beendigung der Gruppe weiterhin stationär blieben. Bei 13 Patienten zeigte sich ein signifikanter Wissenszuwachs $(F=6,513, p=0,025)$. 


\section{Prädiktoren im Prä-Post-Design}

Es stellte sich die Frage, welche Patienten von der zusätzlichen Gruppentherapie profitieren. Bei den klinischen und soziodemografischen Variablen (Schul- und Berufsabschluss, Beschäftigung, Schweregrad der Erkrankung, Geschlecht, Diagnose, Behandlungsstatus zu Gruppenende) wurden aufgrund des Medians Subgruppen gebildet und im Weiteren ANOVAs berechnet. Die Prädiktoren für die Ergebnisvariablen ergaben keine signifikanten Unterschiede.

\section{Diskussion}

Ziel dieser Studie war die Umsetzbarkeit eines kognitivpsychoedukativen Gruppenkonzepts bei stationären depressiven Patienten unter Psychopharmakotherapie. Indikationsspezifische Gruppeninterventionen haben als therapeutisch klar strukturierte und fokussierte Gruppen in den letzten Jahren an Bedeutung gewonnen und sollten einen integralen Bestandteil der Behandlung darstellen [Linden, 2006; Worringen, 2006]. Diese Gruppenintervention kombinierte bewährte Inhalte wie Aufbau positiver Aktivitäten und kognitive Umstrukturierung [Hautzinger, 2003; Lewinsohn et al., 1984] mit Psychoedukation zur Erkrankung, Behandlung und Rückfallprophylaxe. Als psychotherapeutische Wirkfaktoren [Grawe et al., 1994] sind die Vermittlung von Kompetenzen und Einsichten, interaktive Prozesse, Strukturierung und Transparenz zu nennen. Die Patienten können von den Erfahrungen und Bewältigungsstrategien anderer profitieren, die diese oder ähnliche Belastungen überwunden haben.

Die Ein- und Ausschlusskriterien ließen bis auf wenige Ausnahmen alle Komorbiditäten auf Achse I und II zu, so dass die Daten auf die Population stationärer depressiver Patienten generalisierbar erscheinen. 231 Patienten mit überwiegend depressiven Episoden oder rezidivierenden Depressionen nahmen an diesen Gruppen teil. Die Dauer der Intervention und ihre Inhalte wurden an die strukturellen Anforderungen angepasst und sowohl geschlossene als auch offene Gruppen umgesetzt. Anhand der erhobenen Daten lässt sich eine sehr gute Akzeptanz des kognitiv-psychoedukativen Gruppenprogramms feststellen. Die geringe Abbrecherrate (Präsenz bei weniger als 50\% der Sitzungen) von 17,1\% spricht für die Akzeptanz der Gruppe durch die Patienten [z.B. de Jong-Meyer et al., 1996: 27,1\%]. Beim Vergleich mit Studien mit niedrigerer Abbrecherquote [z.B. Backenstraß et al., 2001: 13,3\%; Schramm et al., 2004: kein Abbrecher] gilt es zu berücksichtigen, dass es sich bei der vorliegenden Stichprobe um eine erheblich beeinträchtigte Klientel handelte. Die Rückmeldung der Patienten sprach für eine sehr hohe Behandlungszufriedenheit: $98 \%$ würden diese Gruppenintervention weiterempfehlen. Die Gruppe wurde als informativ, ihre Inhalte als hilfreich und im Alltag umsetzbar sowie ermutigend erlebt. Auch die Befindlichkeit während der Gruppe wurde recht ausgeglichen bewertet. Die Patienten (insbesondere jene, die ambulant teilnahmen) spür- ten den Aufwand für die regelmäßige Teilnahme, nahmen aber dennoch sehr konstant an den Gruppen teil. Dieses Ergebnis entspricht den Erwartungen ausgehend von dem nachgewiesenen hohen Informationsbedarf von Patienten mit depressiven Störungen. Vorbehalte, die Konfrontation mit Symptomen oder Krankheitsverläufen könnte zu einer Symptomverschlechterung beitragen, konnten ausgeräumt werden. Unseres Erachtens sollten belastende Themen (z.B. Symptome, Krankheitsverlauf) mit ressourcenorientierten Inhalten (z.B. Frage, was die Betroffenen bislang als hilfreich erlebt haben) abwechseln. Das strukturierte Vorgehen (Vorgabe des Themas, interaktive Arbeit an der Flipchart: didaktische Zusammenfassung und Beiträge der Teilnehmer, Vertiefung mit Handouts und Arbeitsmaterialien, kurzes Feedback der Teilnehmer am Ende der Sitzung) hilft dem Patienten, seine kognitiven Defizite zu kompensieren. Im weiteren Verlauf wurden die Patienten in einem Interview vor Gruppenbeginn zu ihren Erwartungen und Zielen befragt, um ihnen transparent zu machen, inwiefern sie das Gruppenangebot für ihre Ziele nutzen können.

Die meisten Patienten waren über einen längeren Zeitraum mit verschiedenen Antidepressiva behandelt worden. Die an einer Subgruppe von 41 Patienten erhobenen Ergebnisse zur fremd- und selbstbeurteilten Depressivität sind als ermutigend zu bezeichnen. Die Responseraten in der vorliegenden Stichprobe nach 6-wöchiger Behandlung sind mit denen von Hautzinger und Mitarbeitern [1996] nach 8 Wochen vergleichbar (39 vs. 43,8\%). Die niedrigen Werte in der Selbstbeurteilung (BDI: Response von 24,4\%) sind ein häufig beschriebenes Phänomen in der Depressionsbehandlung: die Einstufung der Patienten stimmt erst nach einer gewissen Zeit mit der Fremdeinstufung überein. Hinsichtlich der hoch signifikanten Verbesserungen gilt es jedoch, die kombinierte Behandlung zu berücksichtigen. Klinische und soziodemografische Variablen erwiesen sich nicht als geeignete Prädiktoren, um ein besonders gutes Abschneiden in der Gruppe voraussagen. Somit zeigte sich, dass die Mehrzahl der Patienten von diesem Angebot profitieren können und höheres Lebensalter ( $>65$ Jahre) kein Ausschlusskriterium sein muss [vgl. Schaub et al., 2001; Hautzinger, 2000].

Diese Studie weist einige methodische Mängel auf. Da die Studie als Implementierungsstudie konzipiert wurde, liegt keine Kontrollgruppe von standardversorgten Patienten vor. Da die Dokumentation unter Routinebedingungen mit relativ geringem zeitlichem Aufwand erfolgte, konnten die Daten nur eingeschränkt erfasst werden. Die Wirkung des psychotherapeutischen Programms ist schwer abschätzbar, da multiple andere Maßnahmen (z.B. Pharmakotherapie, Ergotherapie, Kunsttherapie) stattfanden, die nicht kontrollierbar waren.

Zusammenfassend kann gesagt werden, dass diese Implementierungsstudie trotz der methodischen Einschränkungen zeigt, dass kognitiv-psychoedukative Ansätze zu einer hohen Behandlungszufriedenheit der stationären Gruppenteilnehmer beitragen. Integrative Behandlungskonzepte, die auf verschiedenen Behandlungsprinzipien multiprofessioneller Teams basieren, sind für die 
Behandlung psychisch Kranker wesentlich. Das beschriebene Gruppenkonzept ist mittlerweile fester Bestandteil an der Klinik für Psychiatrie und Psychotherapie der LMU München

\section{Dank}

Unser Dank gilt den PatientInnen, die durch ihre Teilnahme dazu beitrugen, das Behandlungsangebot zu optimieren, Smith Kline Beecham für die finanzielle Unterstützung (1998-2000), für die Konzeptualisierung G. Stotz, J. Graf und der Station C1, für die Durchführung der Gruppen den PsychologInnen im Praktikum J. Arzt, S. Asar, M. Beer, S. Duschek, D. Ernst, M. Fetscher, U. Goldmann, J. Graf, M. Haimerl, S. Kaff, C. Ott, A. Neusser, R. Roeder, U. Schmid, I. Scholler, M. Simon, G. Stotz, Z. Stiftl, G. Unterholzner, B. Walter, B. Wiese, B. Wolf und den ÄrztInnen T. Baghai, P. Dobmeier, S. Ehrentraut, D. Eser, T. Frodl, E. Hofschuster, A. Marcuse, F. Müller-Siecheneder, P. Mickaiel, G. Neundörfer, G. Schmitt, K. Schorr, C. Schüle, U. Wegner. Das Bundesministerium für Bildung und Forschung hat im Rahmen des Kompetenznetzes Depression eine spätere Evaluation des Therapiekonzeptes ermöglicht.

\section{Literatur}

Asberg M, Montgomery SA, Perris C, Schalling D, Sedvall G: A comprehensive psychopathological rating-scale. Acta Psychiatr Scand 1978;271:5-25.

Backenstraß M, Kronmüller KT, Schwarz T, et al: Kognitive Verhaltenstherapie in und mit Gruppen - ein Behandlungsprogramm für depressive Patienten in stationärer Behandlung. Verhaltenstherapie 2001;11: 305-311.

Bandura A: Self-efficacy: toward a unifying theory of behavioral change. Psychol Rev 1977;84:191-215. Bauer M, Whybrow PC, Angst J, Versiani M, Möller HJ: Biologische Behandlung unipolarer depressiver Störungen. Behandlungsleitlinien der World Federation of Societies of Biological Psychiatry (WFSBP). Stuttgart, Wissenschaftliche Verlagsgesellschaft, 2004. Beck AT, Steer RA: Beck Depression Inventory (BDI). San Antonio, Psychological Corporation, 1987. Beck AT, Rush AJ, Shaw BF, Emery G: Kognitive Therapie der Depression. Weinheim, Psychologie Verlags Union, 1994.

Benkert O, Hippius H: Kompendium der Psychiatrischen Pharmakotherapie. Berlin, Springer, 1998 de Jong-Meyer R, Hautzinger M, Rudolf GAE, Strauß W, Frick U: Die Überprüfung der Wirksamkeit einer Kombination von Antidepressiva- und Verhaltenstherapie bei endogen depressiven Patienten: Varianzanalytische Ergebnisse zu den Haupt- und Nebenkriterien des Therapierfolgs. Z Klin Psychol 1996;25(2):93-109. de Jong-Meyer R, Hautzinger M, Kühner C, Schramm E: Leitlinien: Psychotherapie affektiver Störungen. 2005. www.klinische-psychologie-psychotherapie.de. DeRubeis RJ, Hollon SD, Amsterdam JD, Shelton RC, Young PR, Salomon RM, O'Reardon JP, Lovet ML, Gladis MM, Brown LL, Gallop R: Cognitive therapy vs. medication in the treatment of moderate to severe depression. Arch Gen Psychiatry 2005;62:409-416. Deutsche Gesellschaft für Psychiatrie, Psychotherapie und Nervenheilkunde (DGPPN) (Hrsg): Praxisleitlinien in Psychiatrie und Psychotherapie, vol 5. Behandlungsleitlinie Affektive Erkrankungen. Darmstadt, Steinkopff, 2000

Frank E, Kupfer DJ, Perel JM, Cornes C, Jarrett DB, Mallinger AG, et a.: Three-year outcomes for maintenance therapies in recurrent depression. Arch Gen Psychiatry 1990;47:1093-1099.

Gloaguen V, Cottraux J, Cucherat M, Blackburn IM: A meta-analysis of the effects of cognitive therapy in depressed patients. J Affect Disord 1998;49:59-72.

Grawe K, Donati R, Bernauer F: Psychotherapie im Wandel. Von der Konfession zur Profession. Göttingen, Hogrefe, 1994

Hamilton M: Hamilton Depressions Skala; in CIPS (Hrsg): Hamilton Depression Scale (Hamilton Psychiatric Rating Scale for Depression). Internationale Skalen für Psychiatrie, ed 3. Weinheim, Beltz, 1986.

Hautzinger M: Depressionen im Alter. Ein kognitivverhaltenstherapeutisches Gruppenprogramm. Weinheim, Psychologie Verlags Union, 2000

Hautzinger M: Kognitive Verhaltenstherapie bei Depressionen, ed 6. Weinheim, Psychologie Verlags Union, 2003
Hautzinger M, Bailer M, Worall H, Keller F: Beck-Depressions-Inventar (BDI). Bern, Huber, 1995. Hautzinger M, de Jong-Meyer R, Treiber R, Rudolf GAE, Thien U: Wirksamkeit Kognitiver Verhaltenstherapie, Pharmakotherapie und deren Kombination bei nicht-endogen, unipolaren Depressionen. Z Klin Psychol 1996;25(2):130-145.

Hegerl U: Depression und Suizidalität. Verhaltenstherapie 2005;15:6-11.

Hegerl U, Plattner A, Möller HJ: Should combined pharmaco- and psychotherapy be offered to depressed patients? A qualitative review of randomized clinical trials from the 1990s. Eur Arch Psychiatry Clin Neurosci 2004;254:99-107.

Herrle J, Kühner C (Hrsg): Depression bewältigen. Ein kognitiv-verhaltenstherapeutisches Gruppenprogramm nach P.M. Lewinsohn. Weinheim, Psychologie Verlags Union, 1994.

Hollon SD, DeRubeis RJ, Shelton RC, Amsterdam JD, Salomon RM, O'Reardon JP, Lovett ML, Young PR, Haman KI, Brent B, Freeman BB, Gallop R: Prevention of relapse following cognitive therapy vs. medications in moderate to severe depression. Arch Gen Psychiatry 2005;62:417-422.

Huber TJ: Stationäre Depressionsbehandlung. Soll man Psychotherapie und Medikamente kombinieren? Nervenarzt 2005;76:270-277.

Jindal RD, Thase ME: Integrating psychotherapy and pharmacotherapy to improve outcomes among patients with mood disorders. Psychiatr Serv 2003;54: 1484-1490.

Keller MB, McCullough JP, Klein DN, et al: A comparison of nefazodone, the cognitive behavioral-analysis system of psychotherapy, and their combination for the treatment of chronic depression. N Engl J Med 2000;342(20):1462-1470.

Lewinsohn PM, Antonuccio DO, Steinmetz JL, Teri L: The coping with depression course. A psychoeducational intervention for unipolar depression. Eugene, OR, Castalia, 1984.

Linden M: Gruppentherapie. Verhaltenstherapie 2006;16:164-165.

Neumann NU, Schulte RM: Montgomery Asberg Depressions Rating Skala - Deutsche Fassung. Erlangen, Perimed, 1989.

Paykel ES, Scott J, Teasdale JD, et al: Prevention of relapse in residual depression by cognitive therapy. A controlled trial. Arch Gen Psychiatry 1999;56(9): 829-835.

Pitschel-Walz G, Bäuml J, Kissling W: Psychoedukation: Depressionen. Manual zur Leitung von Patienten- und Angehörigengruppen. München, Urban und Fischer, 2003.

Pitschel-Walz G, Bäuml J, Bender W, et al: Psychoeducation and compliance in the treatment of schizophrenia: Results of the Munich Psychosis Information Study. J Clin Psychiatry 2006;67(3):443-52

Risch A, Stangier U: Neuere kognitiv-verhaltenstherapeutische Ansätze zur Rückfallprophylaxe bei rezidivierender Depression. Verhaltenstherapie 2006;16: 275-281.
Schaub A: Feedback Fragebogen; in Bäuml J, PitschelWalz G: Psychoedukation bei schizophrenen Erkrankungen. Stuttgart, Schattauer, 2003, pp 330-333.

Schaub A: Angehörigenarbeit und psychoedukative Patientengruppen in der Therapie affektiver Störungen; in Möller HJ (Hrsg): Handbuch der Therapie psychiatrischer Erkrankungen. Stuttgart, Thieme, 2006, pp 462-473.

Schaub A, Plattner A, Ehrhardt T, Kaschel R: Kognitiv-verhaltenstherapeutische Interventionen bei kognitiven Defiziten und Depressionen im höheren Lebensalter; in Hegerl U, Zaudig M, Möller H-J: Depression und Demenz im Alter. Wien, Springer, 2001, pp 125-150.

Schaub A, Bernhard B, Gauck L: Kognitiv-psychoedukative Therapie bei bipolaren Erkrankungen. Ein Therapiemanual. Göttingen, Hogrefe, 2003.

Schaub A, Roth E, Goldmann U: Kognitiv-psychoedukative Therapie zur Bewältigung von Depressionen Ein Therapiemanual. Göttingen, Hogrefe, 2006.

Schramm E, van Calker D, Berger M: Wirksamkei und Wirkfaktoren der Interpersonellen Psychotherapie in der stationären Depressionsbehandlung - Ergebnisse einer Pilotstudie. Psychother Psychosom Med Psychol 2004;54(2):65-72.

Stuart S, Bowers WA: Cognitive therapy with inpatients. Review and meta-analysis. J Cogn Psychother 1995;9:85-92.

Trautmann-Sponsel RD, Trautmann-Schareck BM Zaudig M: Ein stationäres Gruppenkonzept zur kognitiv-behavioralen Therapie der Depression. Psychotherapie 2000;5(1):114-120.

Vieweg T, Trabert W: Psychoedukation in der Depressionsbehandlung. Ein psychoedukatives Gruppenprogramm im Rahmen stationärer Psychotherapie. Verhaltensther Verhaltensmed 2002;23(4):479-497.

Wolfersdorf M: Depressionsstationen. Konzepte, Erfahrungen, Möglichkeiten heutiger Depressionsbehandlung. Berlin, Springer, 1997.

Wolfersdorf M, Arbeitsgemeinschaft «Suizidalität und Psychiatrisches Krankenhaus»: Suizid im Krankenhaus - Überblick und aktueller Stand zum Suizid während stationärer psychiatrischer Behandlung. Verhaltenstherapie 2005;15:103-109.

World Medical Association declaration of Helsinki: Recommendations guiding physicians in biomedical research involving human subjects JAMA 1997;277: 925-926.

Worringen $\mathrm{U}$ : «Patientenschulung ist ein zentraler Behandlungsbaustein in der medizinischen Rehabilitation». Verhaltenstherapie 2006;16:222-224.

Zielke M: Affektive Störungen und Angststörungen Förderung und Entwicklung antidepressiven Verhaltens in der stationären Behandlung; in Zielke $\mathrm{M}$, Sturm J (Hrsg): Handbuch der stationären Verhaltenstherapie. Weinheim, Psychologie Verlags Union, 1994, pp 473-498. 